\title{
Phonon Softening in Superconducting Diamond
}

\author{
M. Hoesch ${ }^{*}$ T. Fukuda, and J. Mizuki \\ SPring-8, JAEA, 1-1-1 Kouto, Sayo, Hyogo, Japan \\ T. Takenouchi and H. Kawarada \\ School of Science and Engineering, Waseda University, 3-4-1 Okubo, Shinjuku, Tokyo, Japan \\ J.P. Suttel $\left.\right|^{\dagger}$ and S. Tsutsui \\ SPring-8, JASRI, 1-1-1 Kouto, Sayo, Hyogo, Japan \\ A.Q.R. Baron \\ RIKEN, 1-1-1 Kouto, Sayo, Hyogo, Japan and \\ SPring-8, JASRI, 1-1-1 Kouto, Sayo, Hyogo, Japan \\ M. Nagao and Y. Takano \\ National Institute for Materials Science, 1-2-1 Sengen, Tsukuba, Japan
}

(Dated: March 28, 2022)

\begin{abstract}
We observe strong softening of optical phonon modes in superconducting $\left(T_{c}=4.2 \mathrm{~K}\right)$ borondoped diamond near the Brillouin zone center using inelastic x-ray scattering from a CVD-grown highly oriented sample. The magnitude of the softening, and our observation that it becomes stronger approaching zone center, supports theoretical models suggesting a phonon-mediated pairing mechanism via coupling of optical phonon modes to Fermi surfaces around the zone center. The electron-phonon coupling parameter is determined as approximately $\lambda=0.33$.
\end{abstract}

Pure diamond is a wide bandgap insulator. When it is boron-doped beyond the metal-to-insulator transition at $n_{B} \approx 3 \cdot 10^{20} \mathrm{~cm}^{-3}$, it shows superconductivity with transition temperatures $T_{c}$ that are remarkably high for an impurity doped material with low carrier density. Superconductivity was reported both in samples grown by high-pressure-high-temperature synthesis with $T_{c}=4 \mathrm{~K}$ at a B-doping level $n_{B} \approx 5 \cdot 10^{21} \mathrm{~cm}^{-3}[1]$ and in samples grown by $\mu$-wave assisted chemical vapor deposition (CVD) 2, 3. . $T_{c}$ depends on the doping level $n_{B}$ and increases up to $T_{c}^{o n} \approx 11 \mathrm{~K}$ (onset) at the highest doping reported so far [4].

Conventional theory of phonon-mediated superconductivity suggests that an ultrahard material like diamond can have a high $T_{c}$ if the high frequency modes have a strong enough electron-phonon coupling (EPC). The bond-stretching optical modes of diamond around $\omega_{0}=$ $160 \mathrm{meV}$ naturally couple to the covalent bonding states of the diamond valence band. Various ab-initio models have predicted the EPC coupling constant $\lambda$. In the typical doping range between $n=2.5-3$ at\%, values between between $\lambda=0.27$ (virtual crystal approximation VCA [5]) and $\lambda=0.39$ (supercell [6]) are calculated and lead to predicted $T_{c}=0.2-4.4 \mathrm{~K}$ that correspond well to those observed in real samples. The nature of the modes that couple to the free charge carriers is, however, difficult to assess by theory and few experimental data are available. VCA naturally focuses on the optical modes of the diamond lattice that couple due to their bondstretching nature [5, 7]. The $\lambda$ calculated in a supercell also includes localized B-modes, where the coupling is en- hanced due to the enhanced valence band wave functions at the dopant sites 8 .

We have studied the lattice dynamics in a sample of highly B-doped superconducting diamond. For the optical phonon branches, we observe a softening that allows the determination of the momentum-dependent EPC parameter $\lambda(q)$. Similar to inelastic neutron scattering, the technique of inelastic x-ray scattering (IXS) probes the momentum-dependent frequency of the phonon excitations. A strong softening of the optical phonon branches is the fingerprint of a strong EPC. Moreover, the observation of the momentum-dependence of the softening provides information on the phase-space of the coupling. This complements early indications of phonon softening from Raman scattering experiments that probe only zero momentum [9. In the case of high B-doping, Raman scattering shows a strong signal from both lattice as well as electronic excitations that could not be disentangled 10 and thus no quantitative data on the EPC have been gained from the Raman spectra. A recent study of far-infrared and THz-radiation absorption spectra shows one peak of EPC in the region of the optical phonons as well as contributions in a region of lower frequency modes [11.

The electronic structure in the metallic regime is described either by a shift of the Fermi level into the diamond valence band (degenerate metal) or by a purely B-related impurity band or a mixture of both. Experimentally, a Fermi level in the diamond valence band was observed by angle-resolved photoemission spectroscopy (ARPES) 12], but evidence for a persistence of an impu- 
a)

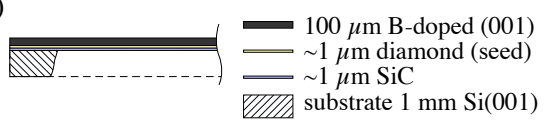

b)

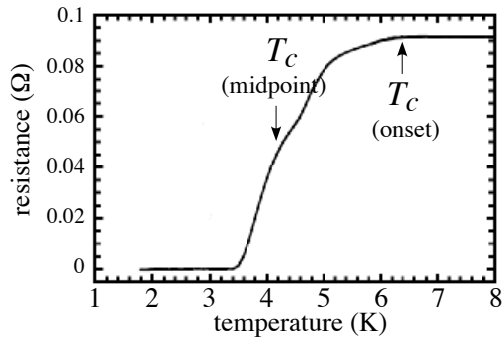

c)

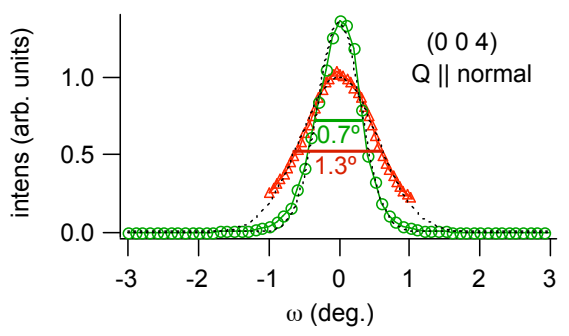

FIG. 1: (a) Schematic view of the $\sim 100 \mu \mathrm{m}$ thick B-doped sample B. (b) Temperature-dependent resistance curve. Above $T_{c}$ the resistance is almost flat with a room temperature resistivity of $\sim 15 \Omega \mathrm{cm}$. (c) X-ray rocking curves of the (004) reflection of sample $\mathrm{B}(\mathrm{\circ})$ and undoped sample $\mathrm{N}(\triangle)$.

rity band close to the top of the valence band maximum was reported by optical spectroscopy [13]. The former scenario would lead to a well-defined "spheroid" Fermi surface (FS) around the $\Gamma$-point. This FS has significant anisotropy due to the covalent bonding states and consists of three concentric sheets. The latter scenario should not lead to a well-defined FS if the dopant atoms occupy random positions in the lattice. Calculations using the coherent phase approximation suggest the formation of a spheroid FS around $\Gamma$ but a smearing of the Fermi wave vector due to impurity scattering [14.

In the presence of a spheroid FS a strong phonon softening is expected at small momentum $q$, where energyconserving phonon scattering is possible within the FS. The softening is gradually reduced to a constant value at increased phonon momentum around the Brillouin zone boundary. This gentle kind of a Kohn anomaly is reminiscent of the EPC in $\mathrm{MgB}_{2}$, where the Cooper pairs form due to a coupling of the electronic $\sigma$-band to the $\mathrm{E}_{2 \mathrm{~g}}$ optical phonons [5, 15, 16.

The disorder in B-doped diamond has only a little direct effect on the lattice dynamics, because the mass difference of B- and C-atoms is small. Like the natural isotope mixture of $\mathrm{C}$-atoms in diamond it would have a significant effect only at doping levels much higher than a few atomic percent 17. In our data we found evidence for a slight disorder-induced change of the Eigenvectors of acoustic modes [18, but no evidence for an EPC of acoustic or localized modes was found. In this letter we discuss the coupling of the optical phonons, where a direct comparison between pure and doped diamond leads to a quantitative determination of the mode-dependent coupling parameter $\lambda$.

The samples were grown by heteroepitaxial CVD deposition on a $\mathrm{Si}(001)$ substrate pre-treated by $\mathrm{SiC}$ to yield a highly ordered (001)-oriented ensemble of coalesced diamond grains [19. A layer of $100 \mu \mathrm{m}$ B-doped diamond at a doping level of $n_{B}=3.8 \cdot 10^{21} \mathrm{~cm}^{-3} \approx 2$ at\% was deposited in $\sim 100$ hours (sample B). The grains are oriented within $1^{\circ}$ as seen in the x-ray rocking curve in Fig. 11. The superconducting transition is rather broad around $T_{c}=4.2 \mathrm{~K}$ (see Fig. 1 $1 \mathrm{~b}$ ). The silicon substrate was removed by chemical etching. A second sample of $100 \mu \mathrm{m}$ of more lightly nitrogen-doped diamond $\left(n_{N}=4 \cdot 10^{18} \mathrm{~cm}^{-3}\right)$ was grown by the same technique (sample N). Nitrogen is introduced to enhance the CVD growth. It does not lead to an electronic doping due to its deep impurity level. Our experiment confirmed the phonon dispersion of sample $\mathrm{N}$ to be identical to pure diamond [20] and we will refer to this reference sample as undoped. The doping levels were determined by secondary ion mass spectroscopy (SIMS).

The experiment was performed at the IXS station of BL35XU 21] at the SPring-8 synchrotron light source. X-rays of $h \nu=15.82 \mathrm{keV}$ were selected with energy resolution of $6.4 \mathrm{meV}$ by use of the $\mathrm{Si}(888)$ reflection in the backscattering monochromator and in the diced spherical analyzers. For each of the two samples 8 positions of the diffractometer arm were measured, which results in 8 spectra precisely on the high symmetry lines $\Gamma$-L and $\Gamma$-X. Additional 21 spectra very close to the high symmetry line at identical positions for undoped and Bdoped diamond were also analyzed. These spectra show only longitudinal phonons (LO) due to the measurement geometry. In addition, a spectrum was acquired at the L-point for the transverse phonons (TA and TO). The energy scale was calibrated for each analyzer using the elastic line position and the optical phonon peak from a pure single crystal diamond sample close to the $\Gamma$-point at $Q=\left(\begin{array}{lll}1.1 & 1.1 & 1.1\end{array}\right)$ [20]. All data were acquired at room temperature because the EPC as such has very weak temperature dependence and can be studied much more conveniently at room temperature than at lower temperature. We remark at this point that even at temperatures below $T_{c}$ no change of the EPC is expected for the optical modes that have frequencies $\omega_{0}$ much larger than the superconducting gap $\omega_{0} \gg 2 \Delta \approx 1 \mathrm{meV}[11,22$.

A selection of measured spectra in the region of the optical phonons is shown in Fig. 2, For small momenta close to the $\Gamma$-point, the peaks are strongly shifted with respect to undoped diamond (extrapolated $-8 \mathrm{meV}$ ). At the Brillouin zone boundary the shift is smaller $(-2 \mathrm{meV})$ both for the X-point and the L-point and for $\mathrm{LO}$ as well as for TO phonons. At the zone boundary the peak shape is 

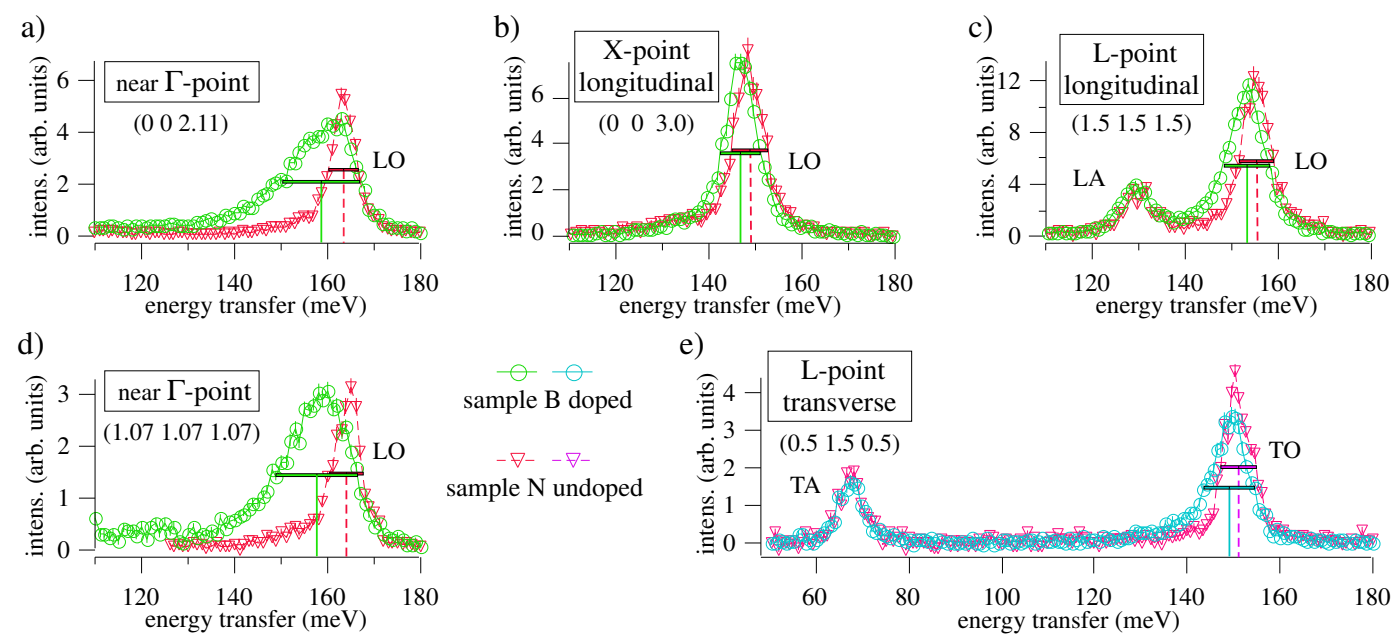

FIG. 2: IXS spectra from undoped sample N $(\nabla)$ and sample B (o) for momentum transfer along the [001]-direction $\Gamma$-X (a and b) and the [111] direction $\Gamma$-L (c and d) in a geometry where only longitudinal phonons contribute, as well as at $Q=(0.51 .50 .5)$ (e) where mostly transverse phonons contribute. The total momentum transfer $Q$ is indicated for each pair of spectra in relative lattice units. The peak position and width at half height is indicated.

given by the resolution function of the IXS spectrometer. Near the zone center the peaks from sample $\mathrm{N}$ are still resolution-limited, while the peaks from sample B are very broad and asymmetric. This asymmetric peak shape is incompatible with a life-time reduction of the phonons in a damped harmonic oscillator theory. More probably

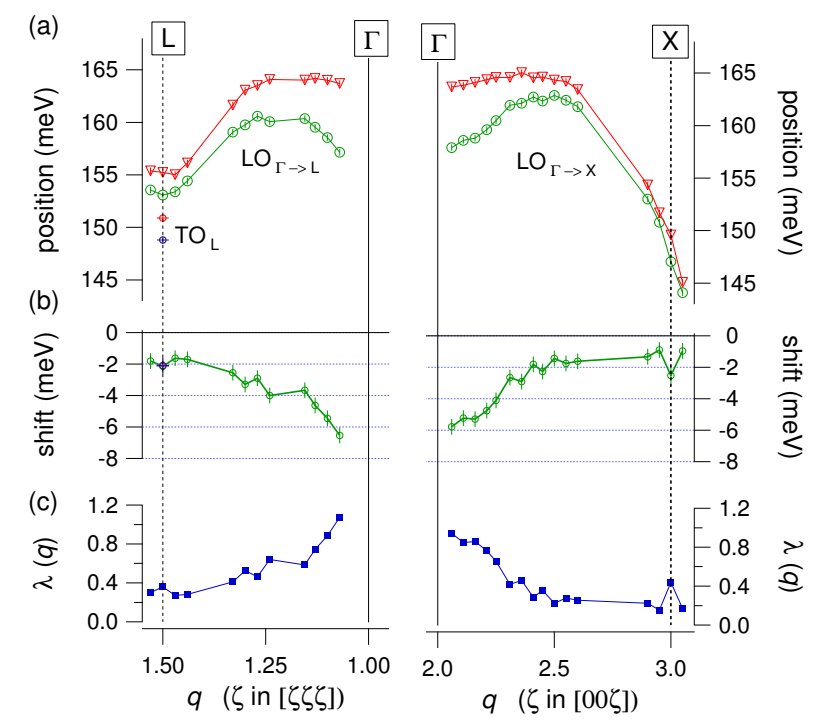

FIG. 3: (a) Dispersion of the optical phonons in sample $\mathrm{N}(\nabla)$ and B (o) as determined by the peak-positions as shown in Fig. 2 In the left-hand side the $\Gamma-\mathrm{L}$ and in the right-hand side the $\Gamma$-X direction is shown. Most data are from the LO phonons, but in the left-hand side at $\mathrm{L}$ the TO phonons are also indicated. (b) Softening curve: peak shift for sample $\mathrm{B}$ with respect to sample $\mathrm{N}$ as a function of momentum. (c) Momentum-dependent EPC parameter $\lambda(q)$ as determined by Eq. (1). it is given by a certain degree of in-homogeneity of the Bcontent across the sample that is also evident in the broad superconducting transition shown in Fig. 1p. The same peak positions and asymmetric shapes were recently also observed in a sample grown by homo-epitaxial CVD [23].

The dispersion shown in Fig. $3 \mathrm{a}$ is determined from the peaks as the arithmetic middle between the two flanks at half height as indicated in Fig. 2. The difference of the dispersions for samples $\mathrm{N}$ and $\mathrm{B}$ is shown in Fig 3 b. Towards $\Gamma$, the peak shift approaches an extrapolated value of $8 \mathrm{meV}$. This value is larger than the one naively derived from the shift in Raman spectra $(<4 \mathrm{meV})$, where the one-phonon line remains barely visible above the background of the lower-energy Fano-feature. The momentum-dependence of the softening is compatible with a gentle Kohn Anomaly in the spheroid FS of a degenerate metal, as the two directions and also longitudinal and transverse phonons soften in the same way. Thus, we find that the phonon softening is purely dependent on the absolute value of the momentum $q$ for the two high symmetry direction we investigated.

To determine the $q$-dependent EPC parameter $\lambda(q)$, the first approach would be to consider the life-time broadening of the phonons and use Allen's formula that connects $\lambda(q)$ to the peak width $\gamma(q)$. In diamond, this approach fails because of the in-homogeneity induced broadening of the LO peaks near $\Gamma$ and their asymmetric line-shape. Instead, we can determine $\lambda(q)$ from the softening from Allen's analysis [24] as

$$
\lambda(q)=\frac{1}{N(0) E_{c}}\left(\frac{\omega_{N}^{2}(q)}{\omega_{B}^{2}(q)}-1\right),
$$

where $E_{c}$ is a cut-off energy identified with the Fermi energy $E_{c}=E_{F}, N(0)$ is the density of states at $E_{F}$ (in 
states $/ \mathrm{eV} /$ cell $)$ and $\omega(q)$ is the measured dispersion for samples $\mathrm{N}$ and B. We take $N(0)$ and $E_{F}$ from VCA calculations for our doping level $(N(0)=0.12$ states $/ \mathrm{eV} /$ cell and $E_{F}=0.62 \mathrm{eV}$ ) [7. The product $N(0) E_{F}$ is proportional to the number of holes per unit cell (in a parabolic band in diamond $N(0) E_{F}=3 n$ ), so that different models of the band dispersion still give only a small error in the determination of $\lambda(q)$. The relevant EPC parameter is the average over the Brillouin zone $\lambda=1 / V_{B Z} \int_{B Z} \lambda(q) d^{3} q$. Assuming isotropic softening we can extrapolate $\lambda(q)$ from either of the two measured momentum directions independently and determine the Brillouin zone average as $\lambda_{\Gamma \mathrm{L}}=0.39$ and $\lambda_{\Gamma \mathrm{X}}=0.27$, respectively. The difference gives an approximate measure of the experimental uncertainty of this analysis. The final value is the average between the two: $\lambda=0.33 \pm 0.06$. The systematic uncertainty due to the theoretical approximations is probably of the same magnitude $( \pm 20 \%)$.

The experimental EPC parmeter lies in the range of theoretical predictions. When inserted into the McMillan relation $T_{c}=\left(\omega_{0} / 1.2\right) \exp \left[-1 /\left(\lambda /(1+\lambda)-\mu^{*}\right)\right]$ using the optical phonon frequency $\omega_{0}=160 \mathrm{meV}$ and a conventional $\mu^{*}=0.12$, a $T_{c}=0.63 \mathrm{~K}$ is derived $\left(T_{c}=3 \mathrm{~K}\right.$ at the higher error limit of $\lambda$ ), which is significantly smaller than the observed $T_{c}=4.2 \mathrm{~K}$. In a direct comparison the measured softening is less strong than the predictions by VCA calculations $(\sim 30 \mathrm{meV}$ at $\Gamma$ and $\sim 6 \mathrm{meV}$ at the L-point [25]). Supercell calculations that provide a more realistic incorporation of the B-atoms into the diamond lattice suffer from ordered-boron artifacts, but the softening reported a a higher doping level of 6.25 at\% (12.5 meV at $\Gamma$ [ 6 ) is in better agreement with our observations. In addition to the optical phonons, these calculations also include coupling to localized B-modes. Thus, although the observed coupling of the optical phonons is strong, there is room for other modes to provide additional EPC.

From our data the determination of $\lambda$ is only possible for those modes that have a direct relation between the doped and the undoped case, i.e. the six phonon branches of the diamond lattice. No coupling was observed for the acoustic modes [18. Also, we have not observed a clear signature from B-derived, localized modes. In the IXS spectra we have, however, observed finite intensity almost throughout the spectral range that is probably related to impurity modes. Our data are thus consistent with a recent optical spectroscopy experiment that found significant contributions to the EPC both in the highfrequency spectral range above $\omega=140 \mathrm{meV}$ as well as in the lower range around $\omega=70 \mathrm{meV}$ [11. The former region corresponds well to the optical phonon coupling reported in this letter. The latter region is also the range of B-B pair vibrations that lead to a very large Raman scattering signal but do not contribute to the EPC [26]. Contributions from single boron localized modes are also predicted around $70 \mathrm{meV}$ 6, 26, matching the analysis of Ref. [1], but so far unaccounted by direct spectroscopy.
In conclusion, we have measured the softening of the optical phonons in diamond. We found a strong softening with a characteristic momentum dependence both along $\Gamma-\mathrm{L}$ and $\Gamma-\mathrm{X}$. This momentum dependence is compatible with coupling to the holes in a spheroid FS as predicted by theory for a Fermi level in the valence band of diamond. We have determined the momentum dependent EPC parameter $\lambda(q)$ for the optical phonons and its average over the Brillouin zone is the mode-specific coupling parameter $\lambda=0.33 \pm 0.06$. Our data thus strongly support an Eliashberg model of the superconductivity in B-doped diamond with coupling through the bond-stretching optical modes that are particularly high in frequency for the ultrahard diamond material.

We would like to thank the Japan Synchrotron Radiation Research Institute (JASRI) for granting beamtime under proposal numbers 2004B0736 and 2005A0596. Dr. Sakaguchi at NIMS characterized the samples by SIMS. Fruitful discussion with M. Tachiki, E. Bustarret, L. Boeri and A. Mirone is gratefully acknowledged. This work was supported by the Grant-in-Aid for Scientific Research on Priority Areas "Invention of anomalous quantum materials" from the MEXT. One of us (MH) would like to thank the Japanese Society for the Promotion of Science (JSPS) for financial support.

* current affiliation ESRF, 6 rue Jules Horowitz, 38000 Grenoble, France; Electronic address: hoesch@esrf.fr

${ }^{\dagger}$ new affiliation Diamond Light Source, Chilton, Didcot, Oxfordshire, England

[1] E.A. Ekimov et al., Nature 428 (2004) 542.

[2] Y. Takano. et al., Appl. Phys. Lett. 85 (2004) 2851

[3] E. Bustarret et al., Phys. Rev. Lett. 93 (2004) 237005.

[4] H. Umezawa et al., cond-mat/0503303

[5] L. Boeri, J. Kortus, O.K. Andersen, Phys. Rev. Lett. 93 (2004) 237002.

[6] H.J. Xiang et al., Phys. Rev. B 70 (2004) 212504.

[7] K.-W. Lee, W. E. Pickett, Phys. Rev. Lett. 93 (2004) 237003.

[8] X. Blase, Ch. Adessi, D. Connetable, Phys. Rev. Lett. 93 (2004) 237004.

[9] K. Okano et al., SPIE Vol. 1275 "Hard Materials in Optics" ed. C.-G. Ribbing (1990) 25.

[10] E. Bustarret, E. Gheeraert, K. Watanabe, Phys. Stat. Sol. (a) 199 (2003) 9.

[11] M. Ortolani et al., Phys. Rev. Lett. 97, 097002 (2006).

[12] T. Yokoya et al., Nature 438 (2005) 647.

[13] D. Wu et al., Phys. Rev. B 73 (2006) 012501.

[14] K.-W. Lee, W. E. Pickett, Phys. Rev. B 73 (2006) 075105.

[15] A. Shukla et al., Phys. Rev. Lett. 90 (2003) 95506.

[16] A.Q.R. Baron et al., Phys. Rev. Lett. 92 (2004) 197004.

[17] F. Widulle, J. Serrano, M. Cardona, Phys. Rev. B 65 (2002) 75206

[18] M. Hoesch et al., Sci. Technol. Adv. Mat. 7 (2006) S31.

[19] H. Kawarada et al., J. Appl. Phys. 81 (1997) 3490.

[20] J. Kulda et al., Phys. Rev. B 66 (2002) 241202(R).

[21] A.Q.R. Baron et al., J. Phys. Chem. Solids 61 (2000) 461. 
[22] K. Ishizaka et al., cond-mat/0604370

[23] M. Hoesch, J. Serrano, M. Krisch, P. Achatz, E. Bustarret, ESRF experimental report IH-HC-957.

[24] P.B. Allen and M.L. Cohen, Phys. Rev. Lett. 29 (1972) 1593.
[25] L.Boeri, J. Kortus, O.K. Andersen, Sci. Technol. Adv. Mat. 7 (2006) S54

[26] E. Bourgeois et al., Phys. Rev. B 74 (2006) 94509. 